\title{
Effect of Short-Term Omega-3 Supplementation on Pulmonary Function after Acute Aerobic Exercise in Athletes with Exercise-Induced Bronchospasm
}

\section{ARTICLE INFO}

\section{Article Type}

Original Research

\section{Authors}

Dehghanianfard M.* MSC, Ghanbarzadeh M. ${ }^{1} P h D$ Habibi A.H. ${ }^{1} P h D$

How to cite this article
Dehghanianfard M,
Ghanbarzadeh M, Habibi A.H.
Effect of Short-Term Omega-3
Supplementation on Pulmonary
Function after Acute Aerobic
Exercise in Athletes with
Exercise-Induced Bronchospasm
Horizon of Medical Sciences.
$2017 ; 23(1): 69-75$.

*Sport Physiology Department, Physical Education \& Sport Science Faculty, Shahid Chamran University of Ahwaz, Ahwaz, Iran ${ }^{1}$ Sport Physiology Department, Physical Education \& Sport Science Faculty, Shahid Chamran University of Ahwaz, Ahwaz, Iran

\section{Correspondence}

Address: Department, Physical Education \& Sport Science Faculty, Shahid Chamran University of Ahwaz, Golestan Street, Ahwaz, Iran Phone: +98 (71) 42233451 Fax: +98 (71) 38256030 mmdehghanianfard@yahoo.com

\section{Article History}

Received: July 4, 2015

Accepted: Septmber 7, 2016

ePublished: January 19, 2017

\section{A B S T R A C T}

Aims Despite many benefits, the sport activities, if they are severe, might affect the athlete's functioning due respiratory system damages called the exercise-induced bronchospasm. The aim of the study was to investigate the short-term side-effects of omega-3 supplementation consumption on the respiratory functioning after severe aerobic activities in the athletes with exercise-induced bronchospasm.

Materials \& Methods In the semi-experimental study, 31 male students with mild exercise-induced bronchospasm were studied in Chamran University of Ahwaz in 2015. The subjects, randomly selected based on the study criteria, were divided into two groups including omega-3 $(n=15)$ and control $(n=16) .1000 m g$ omega- 3 supplementation was daily administrated in omega-3 group for 3 weeks. After an exhaustive activity (Astrand Treadmill Test), the pulmonary functioning was assessed in both group at both pretest and posttest steps. Data was analyzed by SPSS 19 software using dependent $\mathrm{T}$ and covariance tests. Findings 3 -week consumption of omega-3 supplementation did not significantly change the pulmonary indices of persons with exercise-induced bronchospasm ( $p>0.05)$. In addition, control and omega-3 groups were not significantly different in the indices $(p>0.05)$.

Conclusion Daily consumption of $1000 \mathrm{mg}$ omega- 3 supplementation for 3 weeks does not affect the pulmonary functioning of persons with mild exercise-induced bronchospasm.

\section{Keywords Fatty Acids, Omega-3; Exercise-Induced Bronchospasm; Pulmonary Function; Aerobic Exercise}

\section{CIT A T I O N L I NKS}

[1] Effects of dietary omeg-3 fatty acid supplementation on HbA1c, total antioxidant capacity and ... [2] The effects of omega-3 supplementation on ... [3] The effect of omega-3 supplementation on serum prostaglandin E2 female athletes after one ... [4] The prevalence of asthma and exercise-induced bronchoconstriction to ... [5] Misdiagnosis of exercise-induced bronchoconstriction in ... [6] Prevalence of respiratory tract obstruction in professional foreign ... [7] Vitamin C may alleviate exercise-induced ... [8] Inflammatory basis of exercise-induced ... [9] Exercise-induced asthma: Fresh insights and an ... [10] An official American Thoracic Society clinical practice guideline: Exerciseinduced ... [11] Asthma deaths during sports: report of a 7-year ... [12] Protective effect of fish oil ... [13] Effect of 8-week omega-3 supplementation on pulmonary function ... [14] The Effect of Combining Fish Oil and Vitamin C on Airway Inflammation and Hyperpnea-Induced Bronchoconstriction in ... [15] Effect of vitamin c supplementation on aerobic capacity, blood pressure and ... [16] Effect of vitamin C administration on leukocyte vitamin C level and severity of bronchial ... [17] Vitamin D and omega-3 ... [18] Assessment of air way resistance indexes and exercise-induced asthma after asingle session of submaximal incremental ... [19] Exercise-induced bronchospasm in the elite ... [20] Comparison of pulmonary function tests before and after exercise pro and semiprofessional ... [21] Effect of n-3 fatty acids and antioxidants on oxidative stress after ... [22] Exercise-induced bronchoconstriction and atopy in Tunisian ... [23] The prevalence of exercise-induced bronchospasm in soccer player ... [24] The effects of short-term fish oil supplementation on ... [25] The effect of omega-3 fatty acids on bronchial hyperresponsiveness, sputum eosinophilia, and mast cell mediators in ... [26] Pilot study of the effects of n-3 polyunsaturated fatty acids on exhaled nitric oxide in ... [27] Effect of n-3 Polyunsaturated Fatty Acids in Asthma after Low-Dose Allergen ... [28] Effect of dietary intake of omega- 3 and omega- 6 fatty acids on severity of asthma in ... [29] Effect of dietary supplementation ... [30] The mechanism of exercise-induced asthma ... [31] Fish oil supplementation reduces severity of ... [32] Dietary polyunsaturated fatty ... 
كه بهعنوان يك نتيجه از فعاليتهاى ورزشى رخ مى إدهد [5]. تصور

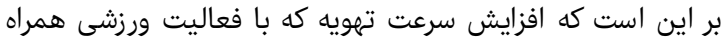

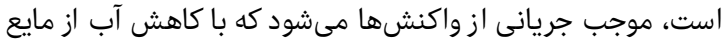
سطح مجارى تنفسى آغاز مىشود[16،

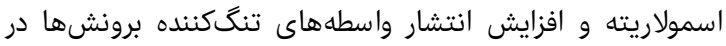

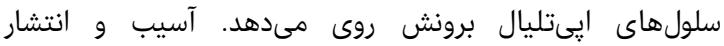

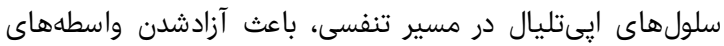

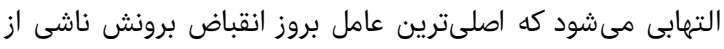

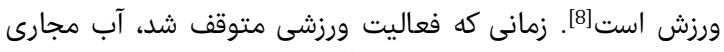

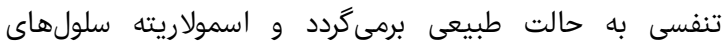

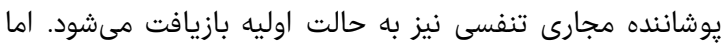

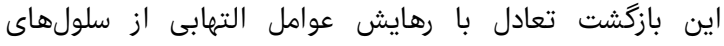

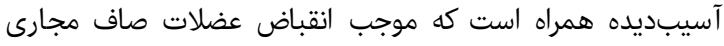

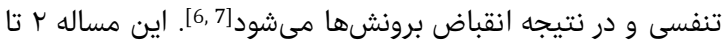

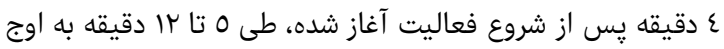

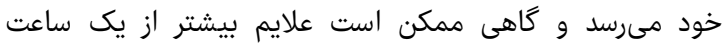

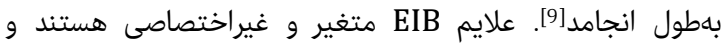

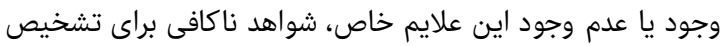
EIB

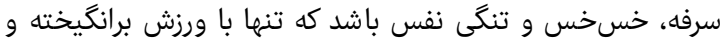

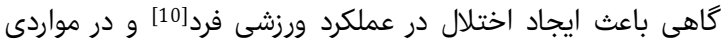

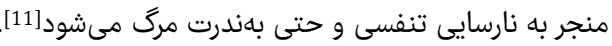

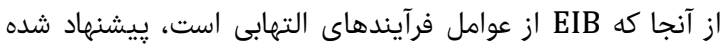

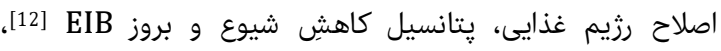

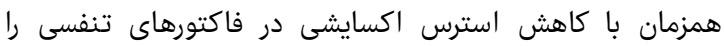

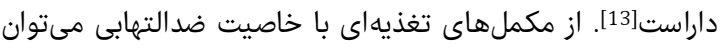

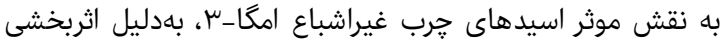

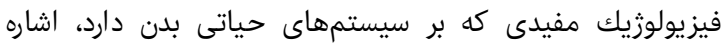

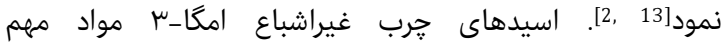

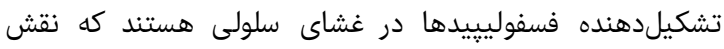

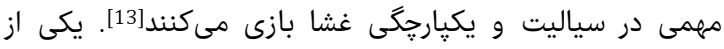

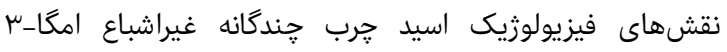

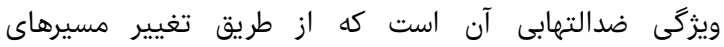

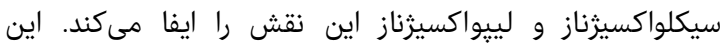

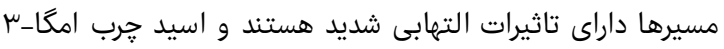

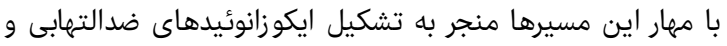

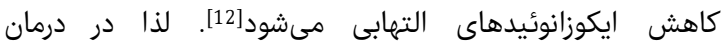

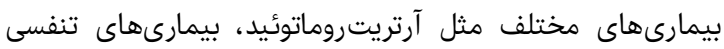

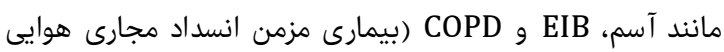

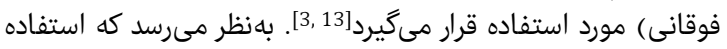

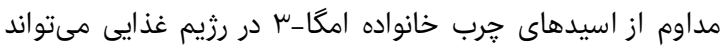

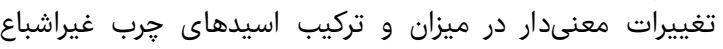

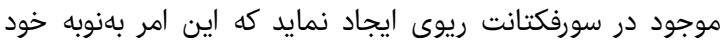

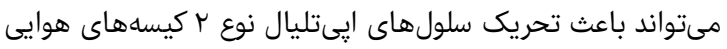

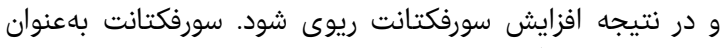

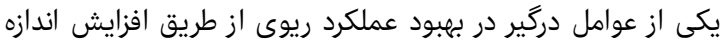

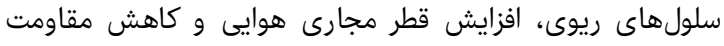

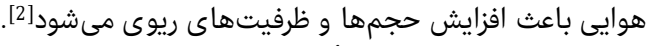

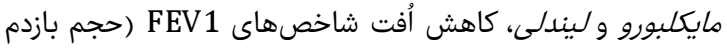

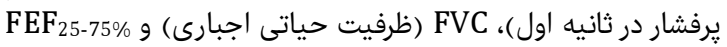

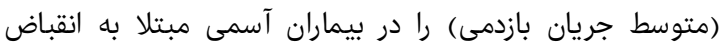

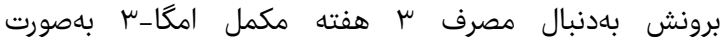

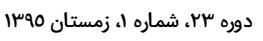

تاثير مصرف كوتاهمدت مكمل امكا_ بر عملكرد

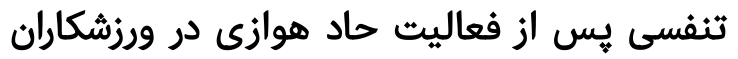
مبتلا به برونكواسياسم ناشى از ورزش هواد هوال

MSc " مريم دهقانيانفرد فروزئ

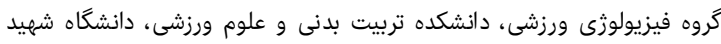
جمران اهواز، اهواز، ايران

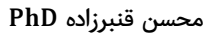

كروه فيزيولوثى ورزشى، داده انشكده تربيت بدنى و و علوم ورزشى، دانشكاه شهيد

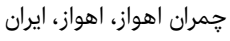

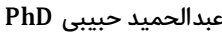

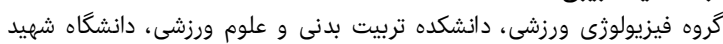
جمران اهواز، اهواز، ايران

جكيده

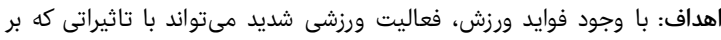

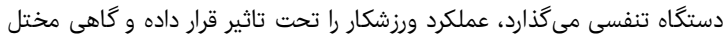

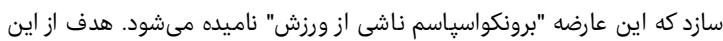

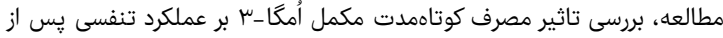

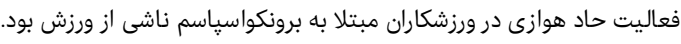

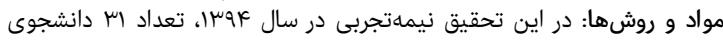

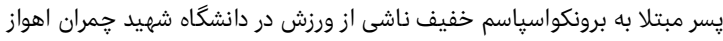

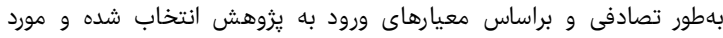

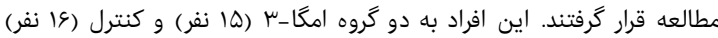

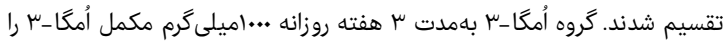

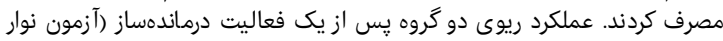

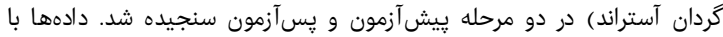

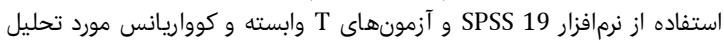

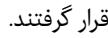

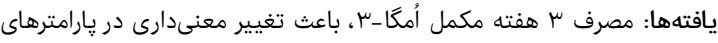

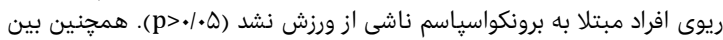

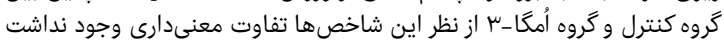
(p>・1.Q)

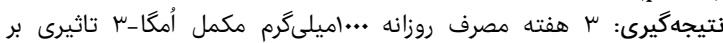

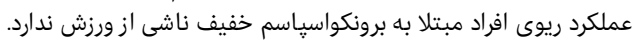

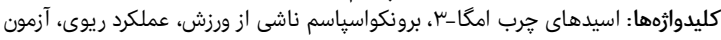

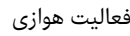

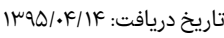

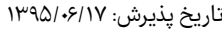
نويسنده مسئول: mmdehghanianfard@yahoo.com

مقدمه

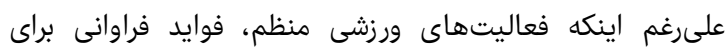

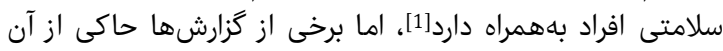

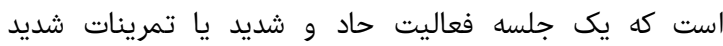

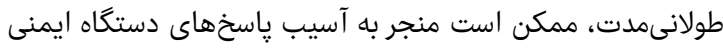

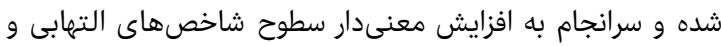

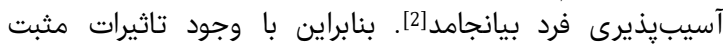

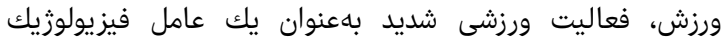

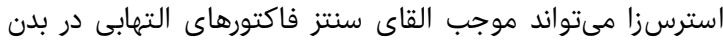

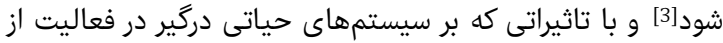

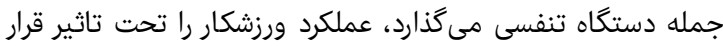
داده و گاهى مختل سازد؛ عارضهاى كه

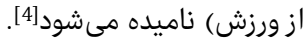

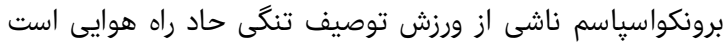
فصل نامه علمى - يُزوهشى افق دانش 


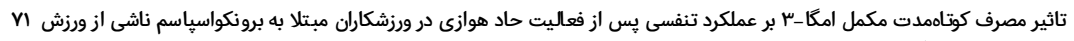

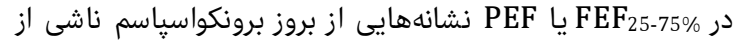
ورزش است[19]. همجنين كاهش

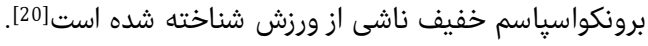

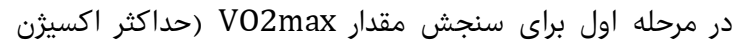

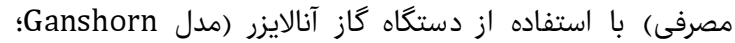

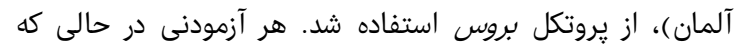

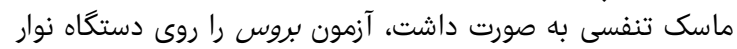

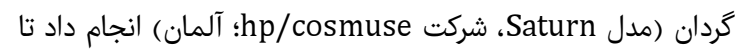

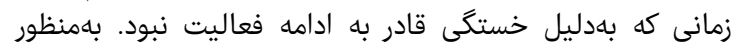

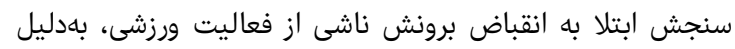

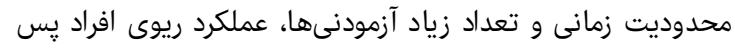

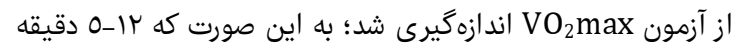

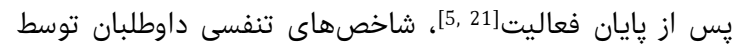

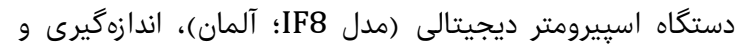

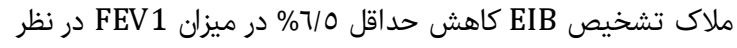
كرفته شد[20].

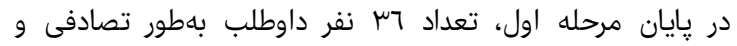

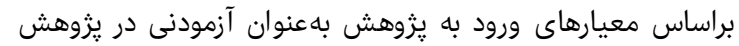

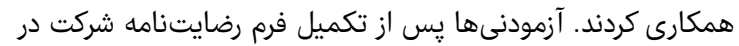

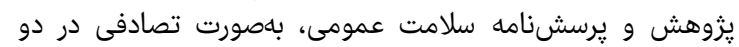

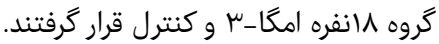

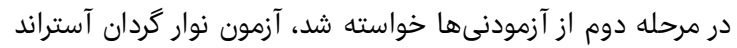

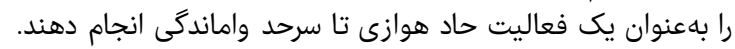

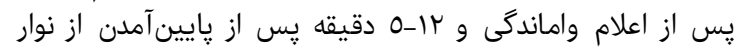

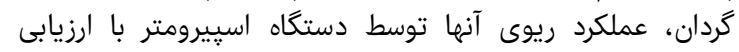

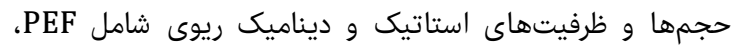
طرح يك و FVC، FEV1 ،FEV1/FVC،MVV

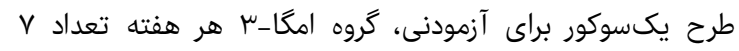

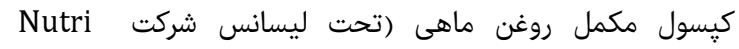
Century Corporation

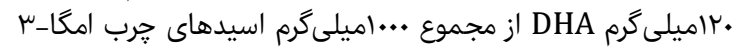

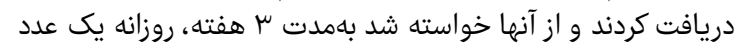

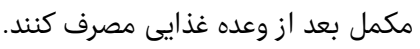

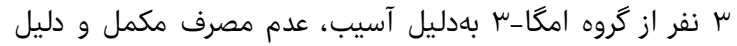

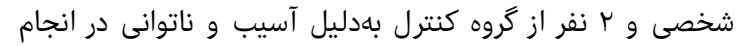

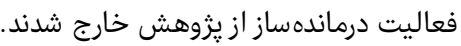

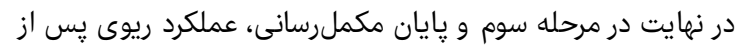

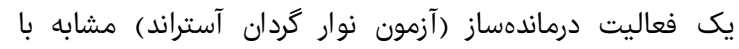

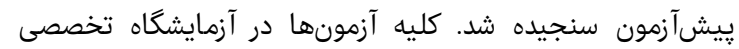

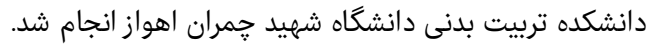

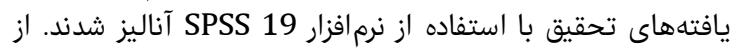

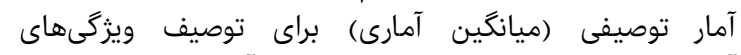

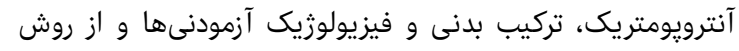

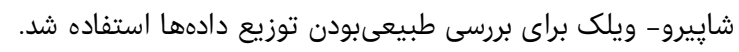

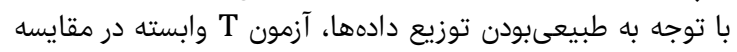

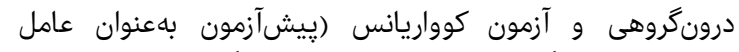

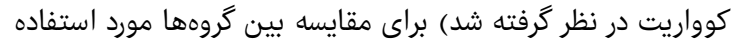

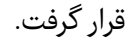

يافتهها نتايج حاصل از دادههاى جمعيتشافناختى دو گروه در جدول ا آمده است.

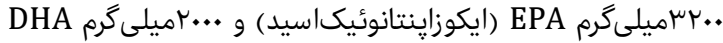

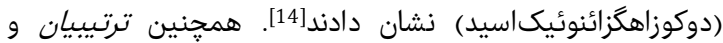

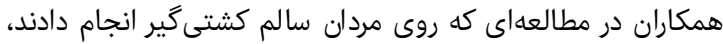

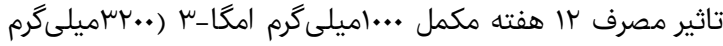

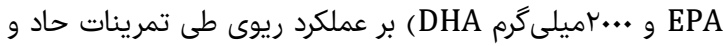

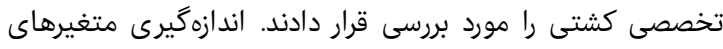

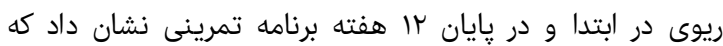

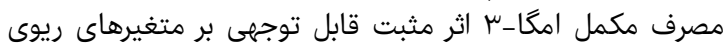
MVV و FEF 25-75\% FEV1 FVC FC

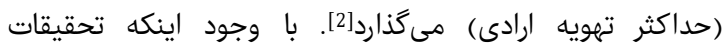

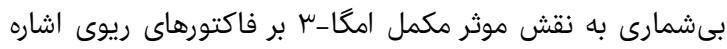

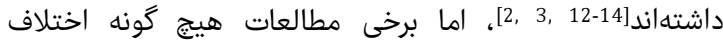

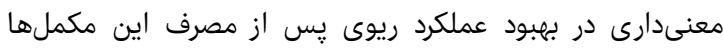

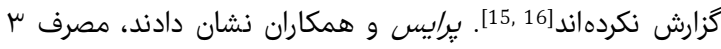

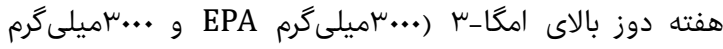

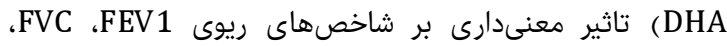

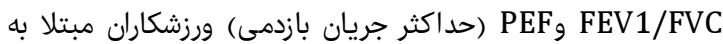

ندارد[17 EIB

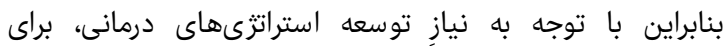

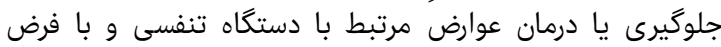

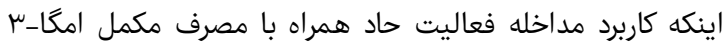

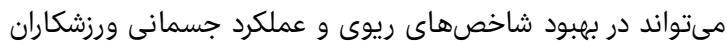

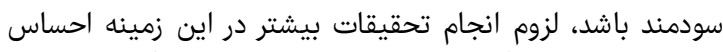

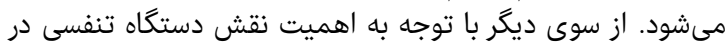

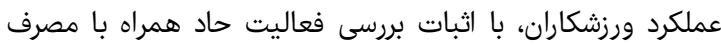

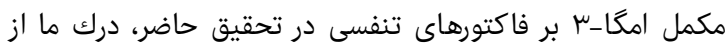

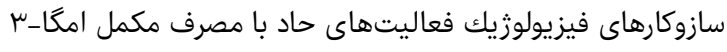

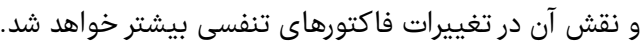

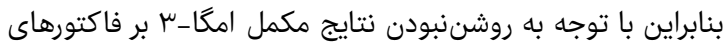

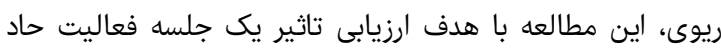

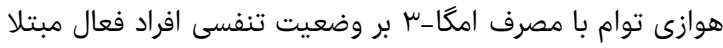

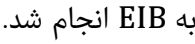

\section{مواد و روشها}

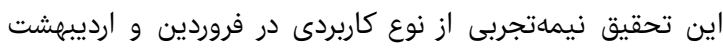

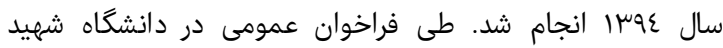

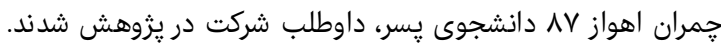

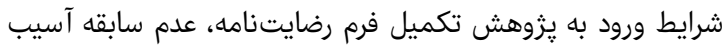

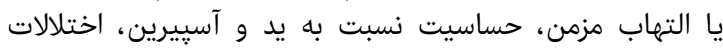

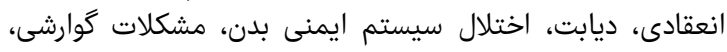

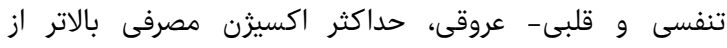

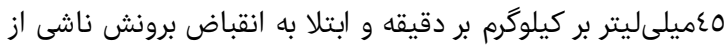

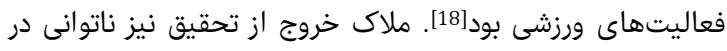

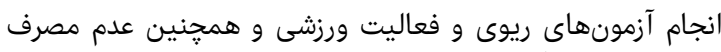

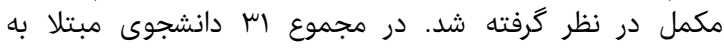

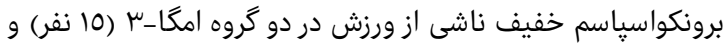
كنترل (17 نفر) مورد مطالعه قرار كرفتند. بهترين روش براى تشخيص EIB مشاهده تغييرات در راههاى مراى

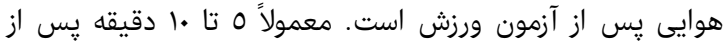

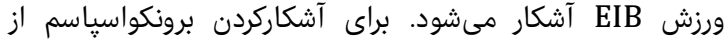

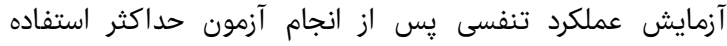

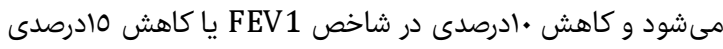


نتايج ما در رابطه با تاثير مصرف مكمل امكا-س بر تغييرات

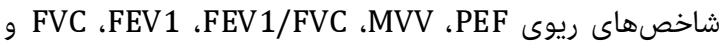

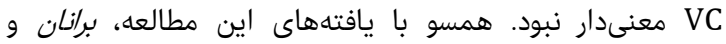

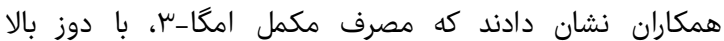

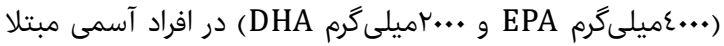

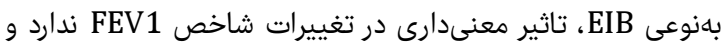

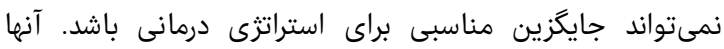

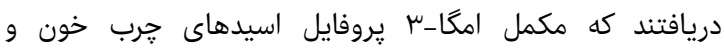

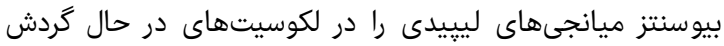

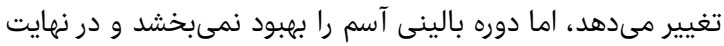

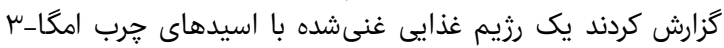

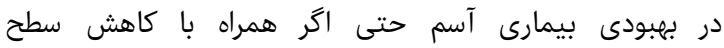

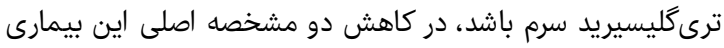

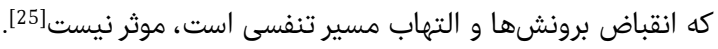

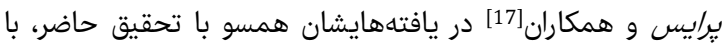

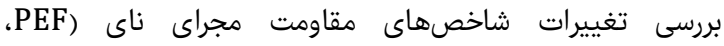
(FVC و FEV1، FEV1/FVC نشان دادند، مصرف س هفتنه مكمل

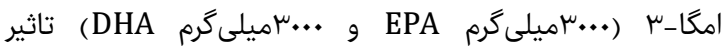

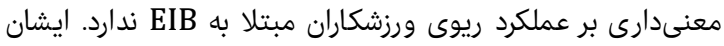
به مصرف دوز مساوى دو اسيد EPA و

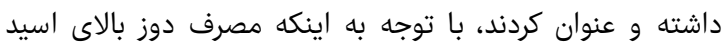

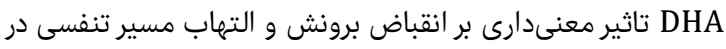

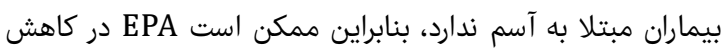

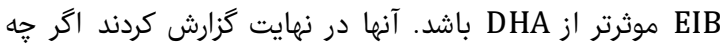

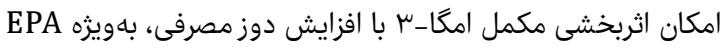

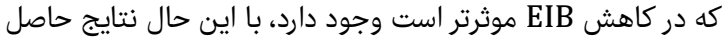

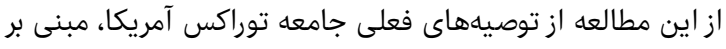

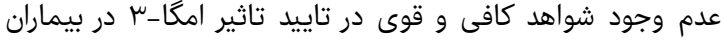

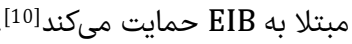

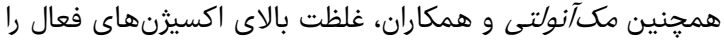

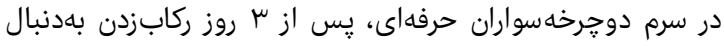

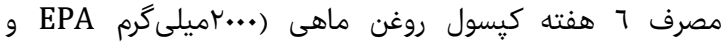
مفيدى •. •ميلى كرم

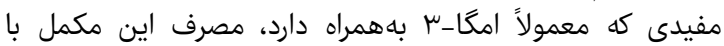

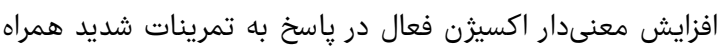

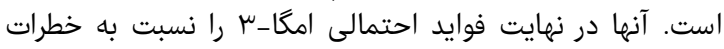

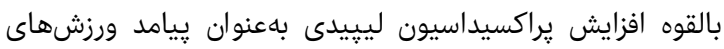
سخت ثابتنشده دانستند [21].

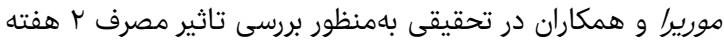

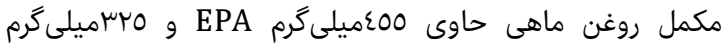

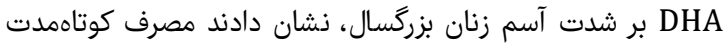

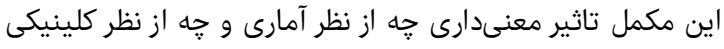

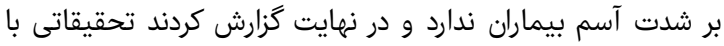

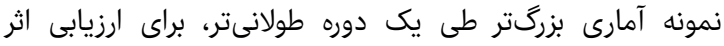

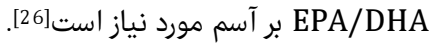
همجنين شوبرت و همكاران نشان دادند مئد مصرف 0 هفتئه كيسول

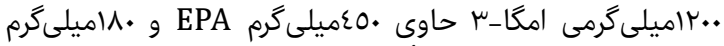

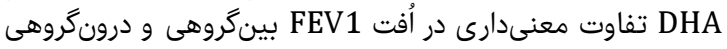

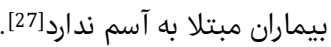

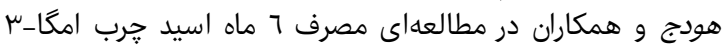

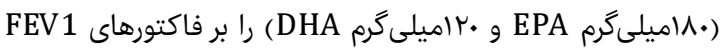

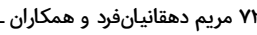

\begin{tabular}{|c|c|}
\hline كروه كنترل (17 نفر) & گروه امكا-س (10 نفر) \\
\hline & سن (سال) \\
\hline \multirow{2}{*}{$r \mid / \cdot . \pm 1 / \cdot \varepsilon$} & $r / V O \pm 1 / 1 r$ \\
\hline & قد (سانتىمتر) \\
\hline \multirow[t]{2}{*}{$|V \varepsilon / r O \pm \sim / \varepsilon|$} & $\mid V V / 0 . \pm 0 / 1 \varepsilon$ \\
\hline & وزن (كيلوگرم) \\
\hline \multirow[t]{2}{*}{$7 r / \mathrm{VO} \pm \mathrm{\Lambda} / \cdot \cdot$} & $79 / \wedge 7 \pm 9 / \mu r$ \\
\hline & نمايه توده بدن (كيلوكرم بر متر مربع) \\
\hline$r / \mu r \pm r / A V$ & $r r / O 1 \pm r / V \mid$ \\
\hline \multicolumn{2}{|c|}{ حداكثر اكسيثن مصرفى (ميلىليتر بر كيلوكرم بر دقيقه) } \\
\hline$\varepsilon \wedge / 77 \pm \varepsilon / 01$ & $\varepsilon q / \varepsilon / \pm r / 7 \varepsilon$ \\
\hline
\end{tabular}

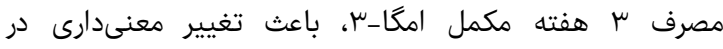

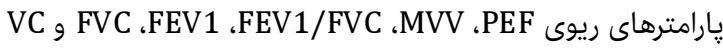

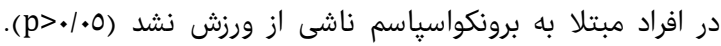

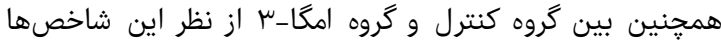

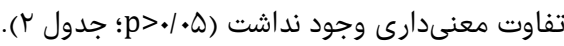

جدول r) ميانكَين آمارى شاخصهاى ريوى در دو گروه در مراحل قبل و بعد از

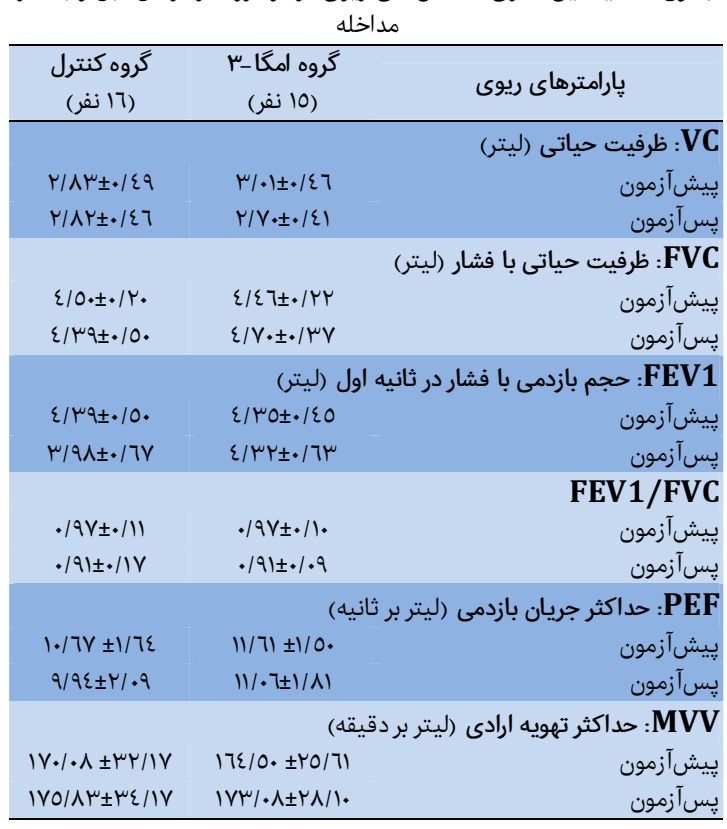

مطالعات كذشته نشان دادهاند عملكرد ريه تحت تاثير فعاليت

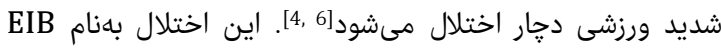

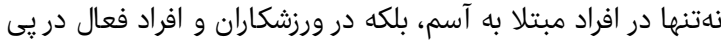

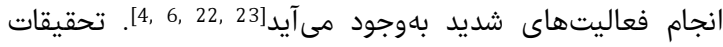

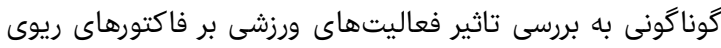

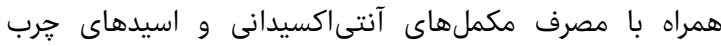
غيراشباع يُرداختهاند]

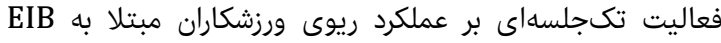

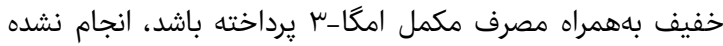

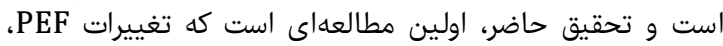

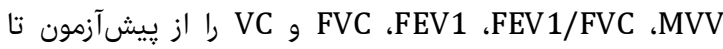

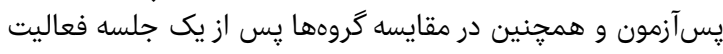

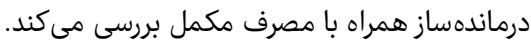




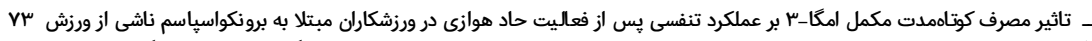

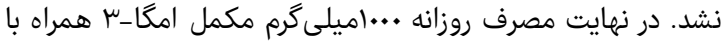

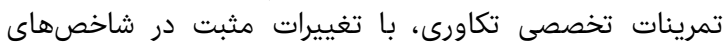

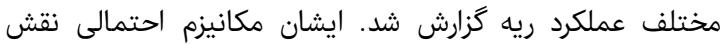

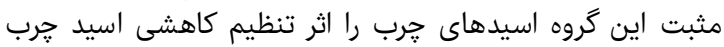

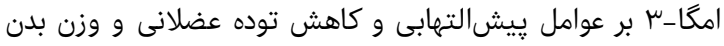

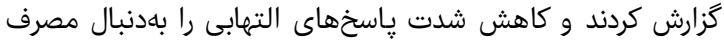
همراه با شركت در تمرينات تكاورى، دليل افزايش

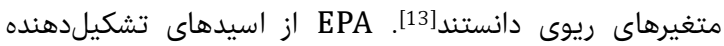

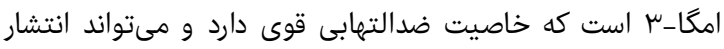

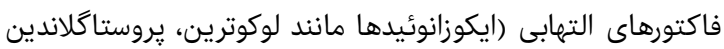

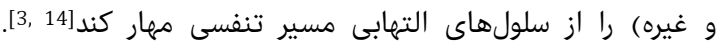

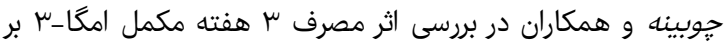

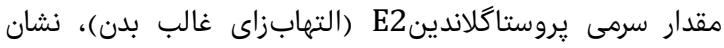

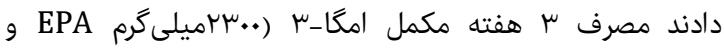

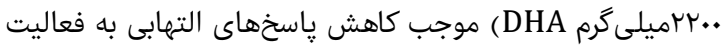

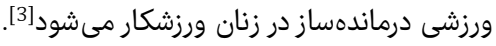

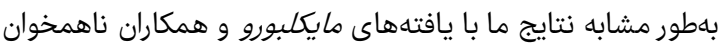

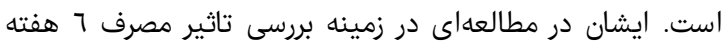

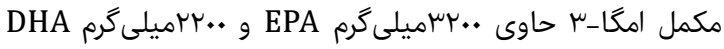

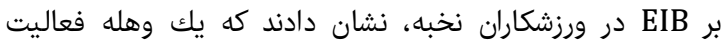

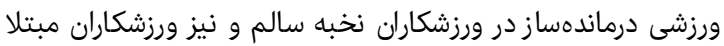

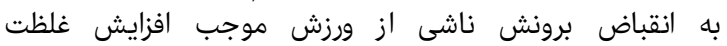

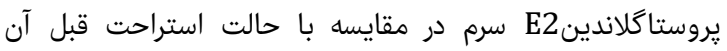

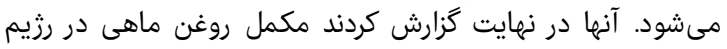

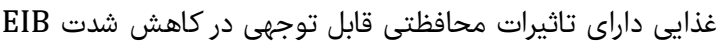

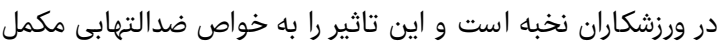

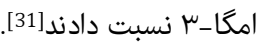

بهطور كلى از جمله دلايل ناهمسويى مطالعات ذكرشده دارئ با نتايج

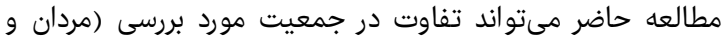

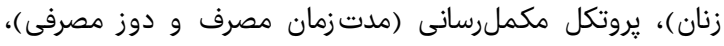

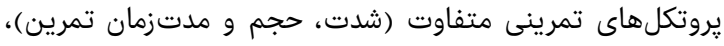

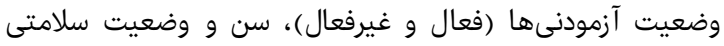

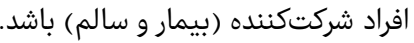

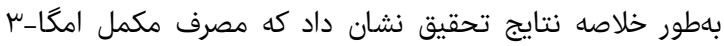

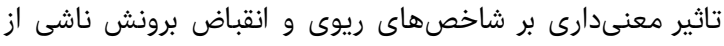

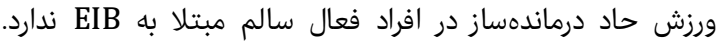

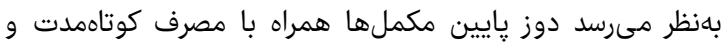

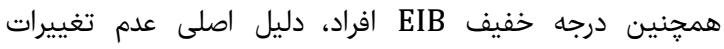

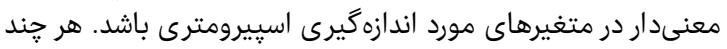

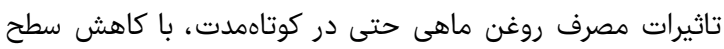

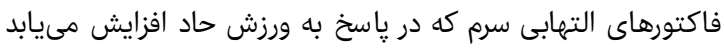

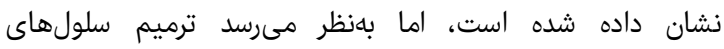

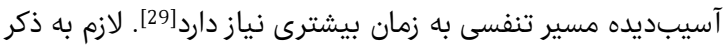

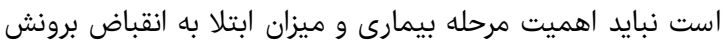

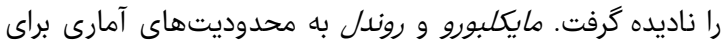

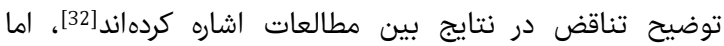

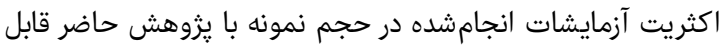

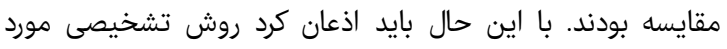

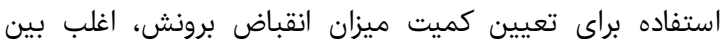

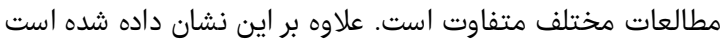

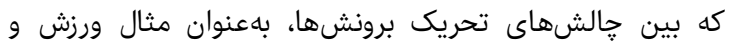

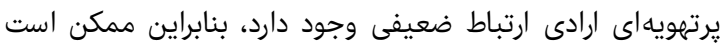

و FVC بدون تاثير گزارش كردند و نتيجه گرفتند حتى با روند رو به

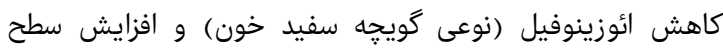

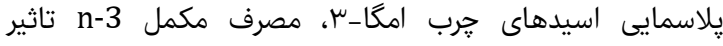

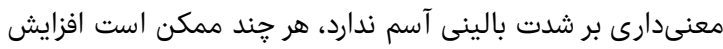

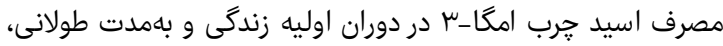

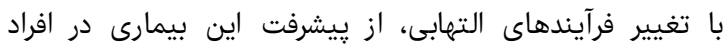
مستعد به آسم جلوكيرى كند [28].

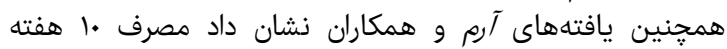

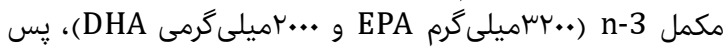

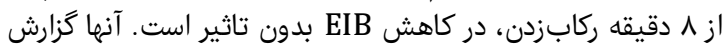

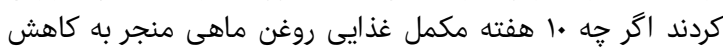

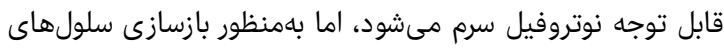

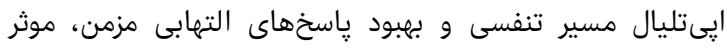

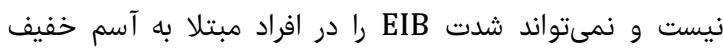

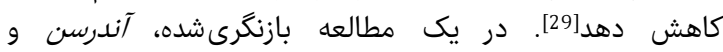

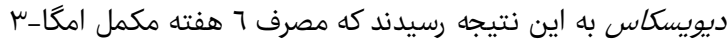
سبب كاهش ياسخ التهابى به يك وهاسيك وهله فعاليت ورزشى بيد بيشينه درف

ورزشكاران نخواهد شد [30].

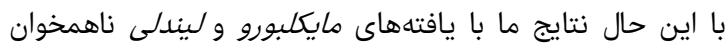

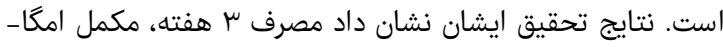

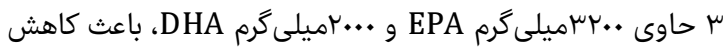

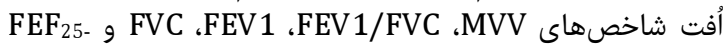
75\% در بيماران آسمى مبتلا به انقباض برونش مى إشود. از نظر

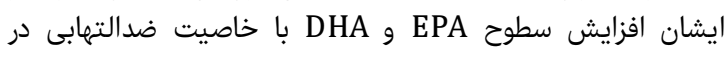

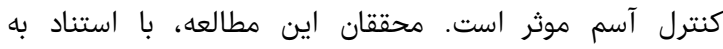

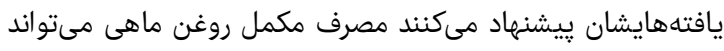

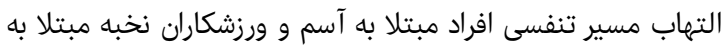
EIB

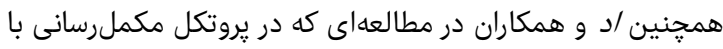

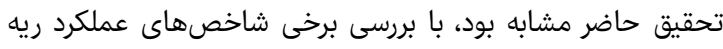

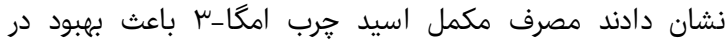

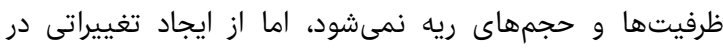

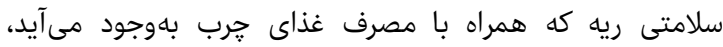

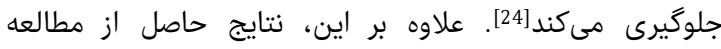

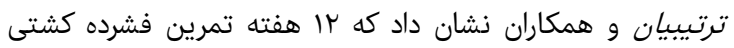

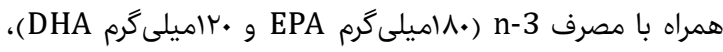

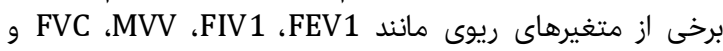
قابل FEF

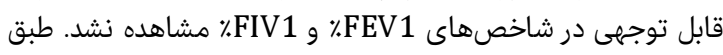

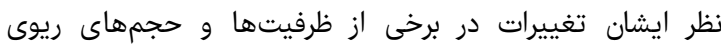

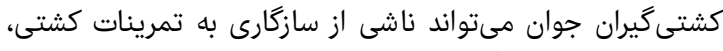

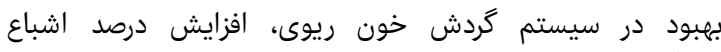

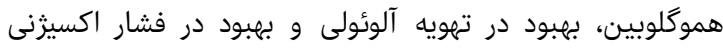

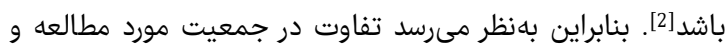

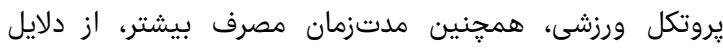
مغايرت باشد.

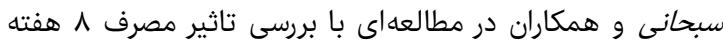

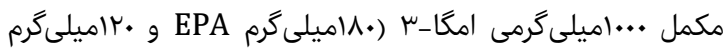

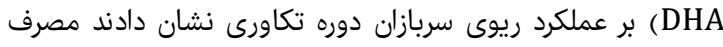

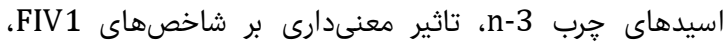

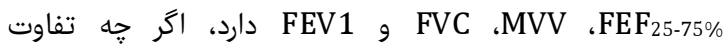
معنىدارى در متغيرهاى PEF و VC و PIF (اوج جريان دمى) ديده

Volume 23, Issue 1, Winter 2017 
of omega-3 supplementation on pulmonary function of young wrestlers during intensive training. J Sci Med Sport. 2010;13(2):281-6.

3- Chubine S, Akbarnejad A, Barjian M, Kordri M. The effect of omega-3 supplementation on serum prostaglandin E2 female athletes after one session exhaustive exercise. Sport Biomotor Sci. 2013;15(4):12133. [Persian]

4- Zunemat Kermani Z, Marefati $H$. The prevalence of asthma and exercise-induced bronchoconstriction to specialized methods in elite endurance cyclists. Olympic. 2014;1(1):37-45. [Persian]

5- Ansley L, Kippelen P, Dickinson J, Hull JH. Misdiagnosis of exercise-induced bronchoconstriction in professional soccer players. Allergy. 2012;67(3):390-5

6- Rabiee MA, GharariArefi R, Ghanbarzadeh M, Habibi A, Marashiyan H. Prevalence of respiratory tract obstruction in professional foreign wrestlers. J Sport Biomotor Sci. 2013;2(8):32-40. [Persian]

7- Hemilä H. Vitamin C may alleviate exercise-induced bronchoconstriction: A meta-analysis. BMJ Open. 2013;3(6):1-7.

8- Hallstrand TS, Moody MW, Wurfel MM, Schwartz LB, Henderson Jr WR, Aitken ML. Inflammatory basis of exercise-induced bronchoconstriction. Am J Respir Crit Care Med. 2005;172(6):679-86.

9- Khajotia R. Exercise-induced asthma: Fresh insights and an overview. Malays Fam Physician. 2008;3(1):21-4.

10- Parsons JP, Hallstrand TS, Mastronarde JG, Kaminsky DA, Rundell KW, Hull JH, et al. An official American Thoracic Society clinical practice guideline: Exerciseinduced bronchoconstriction. Am J Respir Criti Care Med. 2013;187(9):1016-27.

11- Becker JM, Rogers J, Rossini G, Mirchandani H, D'Alonzo GE. Asthma deaths during sports: report of a 7year experience. J Allergy Clin Immunol. 2004;113(2):264-7.

12- Mickleborough TD, Lindley MR, Ionescu AA, Fly AD. Protective effect of fish oil supplementation on exerciseinduced bronchoconstriction in asthma. Chest. 2006;129(1):39-49.

13- Sobhani V, Hajizadeh B, Bazgir B, Kazemipour M, Shamsoddini A, Shakibaey A. Effect of 8-week omega-3 supplementation on pulmonary function during classic army ranger training. Feyz. 2014;17(6):553-60. [Persian] 14- Mickleborough TD, Lindley MR. The Effect of Combining Fish Oil and Vitamin C on Airway Inflammation and Hyperpnea-Induced Bronchoconstriction in Asthma. J Allergy Ther. 2014;5(4):1-10.

15- Choudhuri D, Choudhuri S. Effect of vitamin c supplementation on aerobic capacity, blood pressure and pulmonary functions in young male subjects. Euro J Sports Exerc Sci. 2013;2(2):6-11.

16- Nadi E, Tavakoli F, Zeraati F, Goodarzi MT, Hashemi $\mathrm{SH}$. Effect of vitamin $\mathrm{C}$ administration on leukocyte vitamin $\mathrm{C}$ level and severity of bronchial asthma. Acta Med Iran. 2012;50(4):233-8.

17- Price OJ, Hull JH, Howatson G, Robson-Ansley P, Ansley L. Vitamin D and omega-3 polyunsaturated fatty acid supplementation in athletes with exercise-induced bronchoconstriction: A pilot study. Expert Rev Respir Med. 2015;9(3):369-78.

18- Fatemi R, Ghanbarzadeh M. Assessment of airway resistance indexes and exercise-induced asthma after a single session of submaximal incremental aerobic exercise. J Hum Kinet. 2010;25(1):59-65.

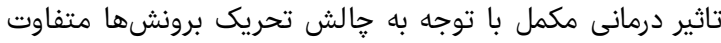

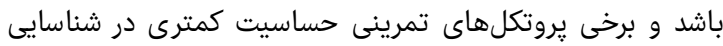

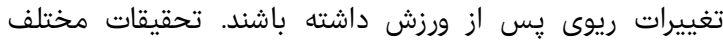

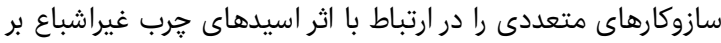

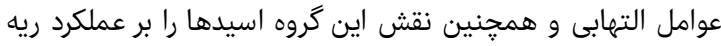

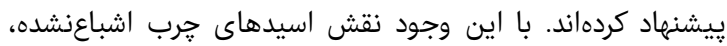

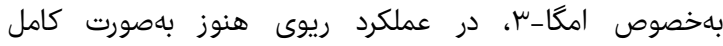
شناختهشده نيست.

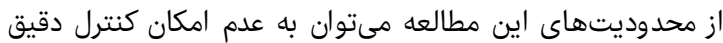

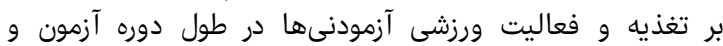

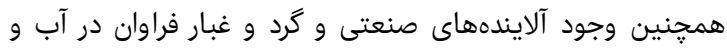

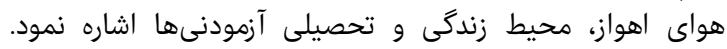

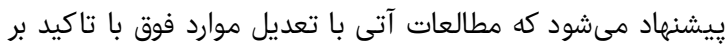

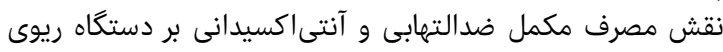
يس ازتمرين ورزشى حاد انجام شود.

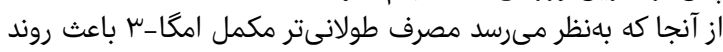

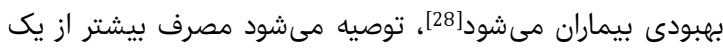

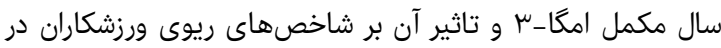

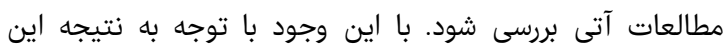

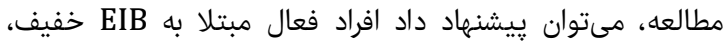

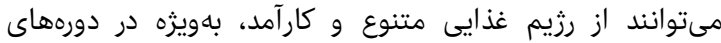
تمرين، بدون استفاده از مكمل هاى غذئن غذايى استفاده كنند.

\section{نتيجه}

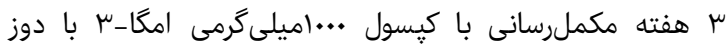

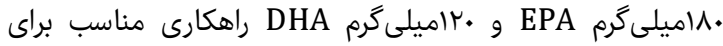
كاهش مقاومت مجراى ناى افراد فعال مبتلا به EPA

نيست.

تشكر و قدردانى: مطالعه حاضر برگرفته از ياياننامه دانشجويى

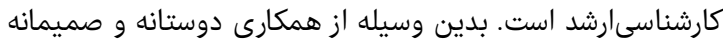

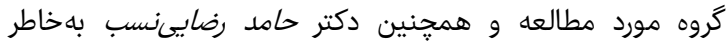

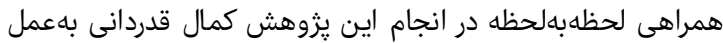
مى آيد. تاييديه اخلاقى: تمام آزمودنىها فرم رضايتنامه شركت در يزوهش

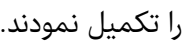
تعارض منافع: هيجز گونه تعارض منافع توسط نويسندگًان بيان

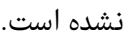

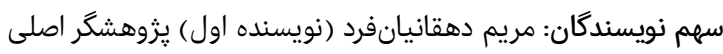

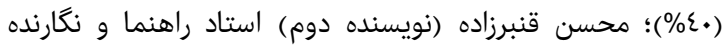

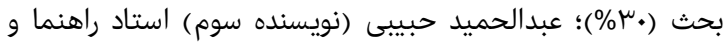

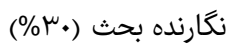

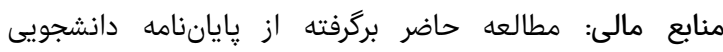
كارشناسىارشد است.

منابع

1- Toorang F, Djazayery A, Jalali M, Eshraghian MR, Farvid M, Pooya SH, et al. Effects of dietary omeg-3 fatty acid supplementation on HbA1c, total antioxidant capacity and superoxide dismutase and catalase activities in type-2 diabetic patients: A randomized clinical trial. Iran J Nutr Sci Food Tech. 2009;3(4):1-8. [Persian]

2- Tartibian B, Hajizadeh Maleki B, Abbasi A. The effects 
VQ تاثير مصرف كوتاهمدت مكمل امكا_س بر عملكرد تنفسى يֶ از فعاليت حاد هوازى در ورزشكاران مبتلا به برونكواسياسم ناشى از ورزش

2015;147(2):397-405.

26- Moreira A, Moreira P, Delgado L, Fonseca J, Teixeira V, Padrao P, et al. Pilot study of the effects of n-3 polyunsaturated fatty acids on exhaled nitric oxide in patients with stable asthma. J Investig Allergol Clin Immunol. 2007;17(5):309-13.

27- Schubert R, Kitz R, Beermann C, Rose MA, Lieb A, Sommerer PC, et al. Effect of n-3 Polyunsaturated Fatty Acids in Asthma after Low-Dose Allergen Challenge. Int Arch Allergy Immunol. 2009;148(4):321-9.

28- Hodge L, Salome CM, Hughes JM, Liu-Brennan D, Rimmer J, Allman M, et al. Effect of dietary intake of omega- 3 and omega- 6 fatty acids on severity of asthma in children. Eur Respir J. 1998;11(2):361-5.

29- Arm JP, Horton CE, Mencia-Huerta JM, House F, Eiser NM, Clark TJ, et al. Effect of dietary supplementation with fish oil lipids on mild asthma. Thorax. 1988;43(2):84-92. 30- Anderson SD, Daviskas E. The mechanism of exercise-induced asthma is... . J Allergy Clin Immunol. 2000;106(3):453-9.

31- Mickleborough TD, Murray RL, Ionescu AA, Lindley MR. Fish oil supplementation reduces severity of exercise-induced bronchoconstriction in elite athletes. Am J Respir Crit Care Med. 2003;168(10):1181-9.

32- Mickleborough TD, Rundell KW. Dietary polyunsaturated fatty acids in asthma-and exerciseinduced bronchoconstriction. Eur J Clin Nutr. 2005;59(12):1335-46.
19- Rundell KW, Jenkinson DM. Exercise-induced bronchospasm in the elite athlete. Sports Med. 2002;32(9):583-600.

20- Ziaee V, AhmadiNejad Z, Farahi A, Movahedi M, Mansoornia M. Comparison of pulmonary function tests before and after exercise pro and semiprofessional basketball. Iran J Basic Med Sci. 2006;9(3):172-7. [Persian]

21- McAnulty SR, Nieman DC, Fox-Rabinovich M, Duran V, McAnulty LS, Henson DA, et al. Effect of n-3 fatty acids and antioxidants on oxidative stress after exercise. Med Sci Sports Exerc. 2010;42(9):1704-11.

22- Sallaoui R, Chamari K, Mossa A, Tabka Z, Chtara M, Feki Y, Amri M. Exercise-induced bronchoconstriction and atopy in Tunisian athletes. BMC Pulm Med. 2009;9(1):8-12.

23- Ziaee V, Yousefi A, Movahedi M, Mehrkhani F, Noorian R. The prevalence of exercise-induced bronchospasm in soccer player children, ages 7 to 16 years. Iran J Allergy Asthma Immunol. 2007;6(1):33-6.

24- Ade CJ, Rosenkranz SK, Harms CA. The effects of short-term fish oil supplementation on pulmonary function and airway inflammation following a high-fat meal. Eur J Appl Physiol. 2014;114(4):675-82.

25- Brannan JD, Bood J, Alkhabaz A, Balgoma D, Otis J, Delin I, et al. The effect of omega-3 fatty acids on bronchial hyperresponsiveness, sputum eosinophilia, and mast cell mediators in asthma. Chest. 